\title{
Comparative effectiveness of pharmacologic and endoscopic interventions for prevention of post-ERCP pancreatitis: a network meta-analysis
}

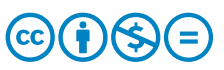

\author{
Authors \\ Basile Njei ${ }^{1,}$, Thomas R. McCarty ${ }^{2,3,}$, Thiruvengadam Muniraj ${ }^{1}$, Prabin Sharma ${ }^{4}$, Priya A. Jamidar ${ }^{1}$, Harry R. \\ Aslanian ${ }^{1}$, Shyam Varadarajulu ${ }^{5}$, Udayakumar Navaneethan ${ }^{5}$
}

Institutions

1 Section of Digestive Diseases, Yale University School of Medicine, New Haven, Connecticut, United States

2 Department of Gastroenterology, Hepatology and Endoscopy, Brigham and Women's Hospital, Boston, Massachusetts, United States

3 Harvard Medical School, Boston, Massachusetts, United States

4 Department of Gastroenterology and Hepatology, Yale New Haven Health-Bridgeport Hospital, Bridgeport, Connecticut, United States

5 Center for Interventional Endoscopy, Florida Hospital, University of Central Florida College of Medicine, Orlando, Florida, United States

submitted 7.2.2019

accepted after revision $\quad 12.8 .2019$

Bibliography

DOI https://doi.org/10.1055/a-1005-6366 |

Endoscopy International Open 2020; 08: E29-E40

(c) Georg Thieme Verlag KG Stuttgart · New York

eISSN 2196-9736

Corresponding author

Basile Njei, MD, MPH, Yale University School of Medicine,

333 Cedar St, New Haven, CT 06520

Fax: +1-860-679-3159

basile.njei@yale.edu

Supplementary material

Online content viewable at:

https://doi.org/10.1055/a-1005-6366
ABSTRACT

Background and study aims While several interventions may decrease risk of post-endoscopic retrograde cholangiopancreatography (ERCP) pancreatitis, it remains unclear whether one strategy is superior to others. The purpose of this study was to compare the effectiveness of pharmacologic and endoscopic interventions to prevent post-ERCP pancreatitis among high-risk patients.

Methods A systematic review was performed to identify randomized controlled trials from PubMed, Embase, Web of Science, and Cochrane database through May 2017. Interventions included: rectal non-steroidal anti-inflammatory drugs (NSAIDs), aggressive hydration with lactated ringer's (LR) solution, and pancreatic stent placement compared to placebo. Only studies with patients at high-risk for post-ERCP pancreatitis were included. Bayesian network meta-analysis was performed and relative ranking of treatments was assessed using surface under the cumulative ranking (SUCRA) probabilities.

Results We identified 29 trials, comprising 7,862 participants comparing four preventive strategies. On network meta-analysis, compared with placebo, rectal NSAIDs $(B=-0.69,95 \% \mathrm{Cl}[-1.18 ;-0.21])$, pancreatic stent $(\mathrm{B}=-1.25,95 \% \mathrm{Cl}[-1.81$ to -0.69$]), \mathrm{LR}(\mathrm{B}=-0.67,95 \% \mathrm{Cl}$ [ -1.20 to -0.13$])$, and combination of $L R$ plus rectal NSAIDs $(B=-1.58 ; 95 \% \mathrm{Cl}[-3.0$ to -0.17$])$, were all associated with a reduced risk of post-ERCP pancreatitis. Pancreatic stent placement had the highest SUCRA probability $(0.81,95 \%$ $\mathrm{Cl}[0.83$ to 0.80$])$ of being ranked the best prophylactic treatment.

Conclusions Based on this network meta-analysis, pancreatic stent placement appears to be the most effective preventive strategy for post-ERCP pancreatitis in high-risk patients.

\footnotetext{
* Drs. Njei and McCarty: These authors contributed equally.
}

\section{Introduction}

Although endoscopic retrograde cholangiopancreatography (ERCP) is commonly performed for diagnosis and management of pancreato-biliary disease, the procedure itself may result in a host of potential adverse events (AEs) - the most common of 
which is post-ERCP pancreatitis [1 - 3]. While the current literature estimates incidence of post-ERCP pancreatitis to be between $3 \%$ to $5 \%$, a recent systematic review including over 2000 high-risk patients who underwent ERCP demonstrated an incidence of $14.7 \%$ with an associated $0.2 \%$ mortality rate [46].

Given this high incidence of post-ERCP pancreatitis in association with substantial morbidity, mortality, and healthcare costs of $\$ 200$ million annually in the United States, it is not surprising that several preventive strategies, both pharmacologic and endoscopic, have been employed [7]. Currently, there are several studies evaluating a variety of pharmacologic prophylaxes for post-ERCP pancreatitis in high-risk patients that include administration of rectal non-steroidal anti-inflammatory drugs (NSAIDs) or aggressive hydration with lactated ringer's solution (LR) [8-13]. In addition, several studies have demonstrated the efficacy of prophylactic pancreatic stent placement in reducing the rate of post-ERCP pancreatitis and more importantly in reducing the risk of severe post-ERCP pancreatitis [14-16].

However, due to paucity of data, it remains unclear whether pharmacologic, endoscopic, or a combination of both approaches is the preferred strategy to prevent post-ERCP pancreatitis. While previous direct pairwise meta-analyses have attempted to answer this question about prevention of post-ERCP pancreatitis, these types of studies are only able to provide partial information due to inherent design limitations. The primary aim of this study is to design a network meta-analysis, simultaneously analyzing direct and indirect evidence, to compare the effectiveness of pharmacologic and endoscopic treatment approaches to prevent post-ERCP pancreatitis.

\section{Methods}

\section{Study design}

This systematic review was performed according to the Preferred Reporting Items for Systematic Reviews and Meta-Analyses (PRISMA) statement outline for reporting systematic reviews and meta-analyses and was conducted following a priori established protocol $[17,18]$. We utilized a network meta-analysis and Bayesian framework to combine direct and indirect evidence comparing the relative efficacy of pharmacologic and endoscopic prophylaxis treatments for post-ERCP pancreatitis. This method was chosen to compare multiple interventions and synthesize evidence across a network of randomized controlled trials $[19,20]$. In brief, this method allows for simultaneous analysis of direct evidence from comparator trials as well as indirect evidence from different treatments compared to a common comparator (i.e., placebo or no intervention) [21].

\section{Selection criteria and study outcomes}

Only randomized controlled trials for high-risk patients were included in this network meta-analysis. High-risk patients were defined in randomized controlled trials by procedure-related factors such as difficult cannulation, pancreatic duct injection, pancreatic sphincterotomy, and pre-cut sphincterotomy in ad- dition to patient-associated factors including female sex, those with sphincter of Oddi dysfunction, and patients with recurrent pancreatitis or prior history of post-ERCP pancreatitis [22-24]. Only trials involving high-risk patients were included.

Included studies involved adult patients (age $\geq 18$ years) who underwent ERCP involving use of rectal NSAIDs (i.e., indomethacin or diclofenac), aggressive hydration with LR solution (defined as $\geq 3 \mathrm{~mL} / \mathrm{kg} / \mathrm{h}$ during the procedure and post-procedure intravenous bolus or high-rate infusion), prophylactic pancreatic stent placement, or placebo. Studies evaluating these treatments alone or in combination as well as studies evaluating direct comparator studies to placebo were included. Observational studies and trials evaluating prevention or reduction of post-ERCP pancreatitis were excluded. Post-ERCP pancreatitis was defined by the consensus criteria as a clinical syndrome consistent with pancreatitis as typical epigastric abdominal pain with an amylase or lipase level at least three times [25].

\section{Data sources and literature search strategy}

Two authors (BN and TRM) independently conducted a comprehensive search of the literature to identify articles that examined multiple electronic databases. Systematic searches of PubMed, EMBASE, Web of Science, the Cochrane Library databases, and major annual gastroenterology conference proceedings were performed from inception through May 31, 2017. Published abstract proceedings were extracted from major gastrointestinal meetings from January 2010 to May 2017. Scientific meetings included Digestive Disease Week (DDW), United European Gastroenterology Week (UEGW), and the American College of Gastroenterology (ACG) annual meeting.

Search terms included: "post-endoscopic retrograde cholangiopancreatography (ERCP) pancreatitis," "rectal non-steroidal anti-inflammatory drugs (NSAIDs)," "lactated ringer's (LR)," "prophylactic pancreatic stent," and "post-ERCP pancreatitis prophylaxis." All relevant articles irrespective of language, year of publication, type of publication, or publication status were included. Titles and abstracts of all potentially relevant studies were screened for eligibility. Reference lists of studies of interest were then manually reviewed for additional articles with additional references acquired through cross-checking bibliographies of retrieved full-text papers. Any differences were resolved by mutual agreement and in consultation with the third reviewer (UN). In the case of studies with incomplete information, contact was attempted with the principal authors to obtain additional data.

\section{Data abstraction and quality assessment}

Study data were abstracted by two authors (BN and TRM) independently. Risk of bias of individual studies was evaluated and assessed in the context of the primary outcome (i.e., development of post-ERCP pancreatitis) using the Cochrane Risk of Bias assessment tool [26]. The GRADE approach to rate the quality of evidence of estimates derived from this network meta-analysis was also performed [27, 28]. Direct evidence from randomized controlled trials was initially identified as high quality; however, it could be down-graded based on risk of bias, indirectness, imprecision, heterogeneity, or publication bias. Rating 
of indirect estimates began at the lowest rating of the two pairwise estimates that contribute as first-order loops to the indirect estimate but could be down-graded further for imprecision or intransitivity. If direct and indirect estimates were similar (i.e., coherent), then the higher of their rating was assigned to the network meta-analysis estimates.

\section{Statistical analysis}

Direct meta-analysis was performed using DerSimonian and Laird random effects model to estimate pooled odds ratio (OR) and $95 \%$ confidence interval $(\mathrm{Cl})$ [29]. Multivariate randomeffects meta-regression was utilized to present results using the Stata mvmeta command extension [30]. Heterogeneity was assessed using $\mathrm{I}^{2}$ statistic with values $>50 \%$ indicating substantial heterogeneity [31]. With regard to publication bias, funnel plot asymmetry and Egger's regression test were performed as well [32]. Direct comparisons were performed using RevMan v5.3 (Cochrane Collaboration, Copenhagen, Denmark) [33].

Indirect comparisons using a fixed effects Bayesian network meta-analysis was performed using a Markov chain Monte Carlo method in WinBUGS statistical analysis program version 1.4.4 (MRC Biostatistics Unit, Institute of Public Health, Cambridge, UK) and Network Plots were created in Stata SE-13 [31,34]. The Bayesian meta-analysis approach offers greater flexibility for the use of more complex models and different outcome types, thereby enabling the simultaneous comparison of all treatment options [35]. Comparative effectiveness of any two treatments were modeled as a function of each treatment in relation to the reference treatment (i. e., placebo or no intervention) to assume consistency of treatment effects across all included randomized controlled trials. This thereby ensures the direct and indirect effect estimates are the same effects.

With regard to network consistency, direct and indirect estimates were evaluated using a node-splitting technique. Posterior distribution of all parameters was estimated using non-informative priors to limit inference to data derived from the trials. No assumptions about efficacy of rectal NSAIDs, aggressive resuscitation with $L R$, or prophylactic pancreatic stent placement from data external to the trials was included in this systematic review and network meta-analysis.

We used the Markov chain Monte Carlo method to obtain pooled effect sizes [35]. All chains were run with 50,000 simulated draws after a burn-in of 5,000 iterations. The median of the posterior distribution based upon 50,000 simulations was obtained using the $2.5^{\text {th }}$ and $97.5^{\text {th }}$ percentiles, after adjusted for multiple arms. Risk of post-ERCP pancreatitis was reported using the estimate of the beta coefficient and calculated the $95 \%$ credible interval.

Information on the relative effects was converted into a probability that a treatment was best, (i. e., first-best, secondbest, etc) and into a ranking for each treatment, called the surface under the cumulative ranking curve (SUCRA) [36, 37]. A SUCRA value of 1.0 guarantees when a treatment is certainly the best and 0 when a treatment is certainly the worst. SUCRA values enable overall ranking of treatments for a particular outcome. SUCRA simplifies the information about the effect of each treatment into a single number, thereby assisting the decision-making process.

\section{Sensitivity analysis}

Sensitivity analyses were also performed to assess the robustness and generalizability of our findings. Sensitivity analysis was performed for high-quality studies as determined by GRADE assessment. Low-quality studies were excluded. Additional sensitivity analysis was performed for full-text manuscripts. Abstracts that did not result in full manuscript publication were excluded.

\section{Results}

Based on previously discussed literature search criteria, a total of 29 trials were included in this study - comprising 7,862 participants comparting four preventive strategies for post-ERCP pancreatitis. A PRISMA flow chart of search results is shown in - Fig.1. A network plot of network meta-analysis comparisons of included studies is highlighted in $>$ Fig. 2.

\section{Characteristics of included studies}

The primary outcome of post-ERCP pancreatitis was reported in all studies ( $\vee$ Table 1 ). Thirteen studies, including 5,955 patients with rectal NSAID use for prevention of post-ERCP pancreatitis were included [8-10,17,38-46]. All but one study (abstract) were fully published manuscripts [45]. Eight studies involving 754 patients evaluated use of aggressive hydration with LR solution in the prophylaxis of post-ERCP pancreatitis [11-13,47-51]. Four studies were fully published manuscripts, three studies were major gastroenterology meeting abstracts, and a final study was a RCT identified through ClinicalTrials.gov. One of the fully published manuscripts was a doubleblinded, placebo-controlled trial that included both LR solution independently, as well as in combination with rectal NSAID use $(n=96)$ [51]. Nine studies, including a total of 1057 participants, evaluated use of prophylactic pancreatic stent placement [14-16, 52-57].

\section{Quality assessment}

GRADE assessment was performed for all included studies. These review authors' judgements about each risk of bias item for each included study is highlighted in Supplemental Fig. 1. A risk of bias summary graph is also available in Supplemental Fig. 2.

\section{Direct treatment comparisons}

For direct treatment comparisons, all pharmacologic and endoscopic modalities were compared to placebo or no intervention (Supplemental Table1). Compared to placebo, use of rectal NSAID therapy significantly reduced odds of post-ERCP pancreatitis by $49 \%(\mathrm{OR}=0.51,95 \% \mathrm{Cl}[0.33$ to 0.77$])$ ( $\mathbf{F i g . 3}$ ). With regard to the effect of aggressive LR solution, this strategy was also effective in significantly reducing post-ERCP pancreatitis by $52 \%(\mathrm{OR}=0.48,95 \% \mathrm{Cl}[0.24$ to 0.97$])$ ( Fig. 4). When these two modalities were combined and compared to placebo, use of rectal NSAID and aggressive LR solution demonstrated 


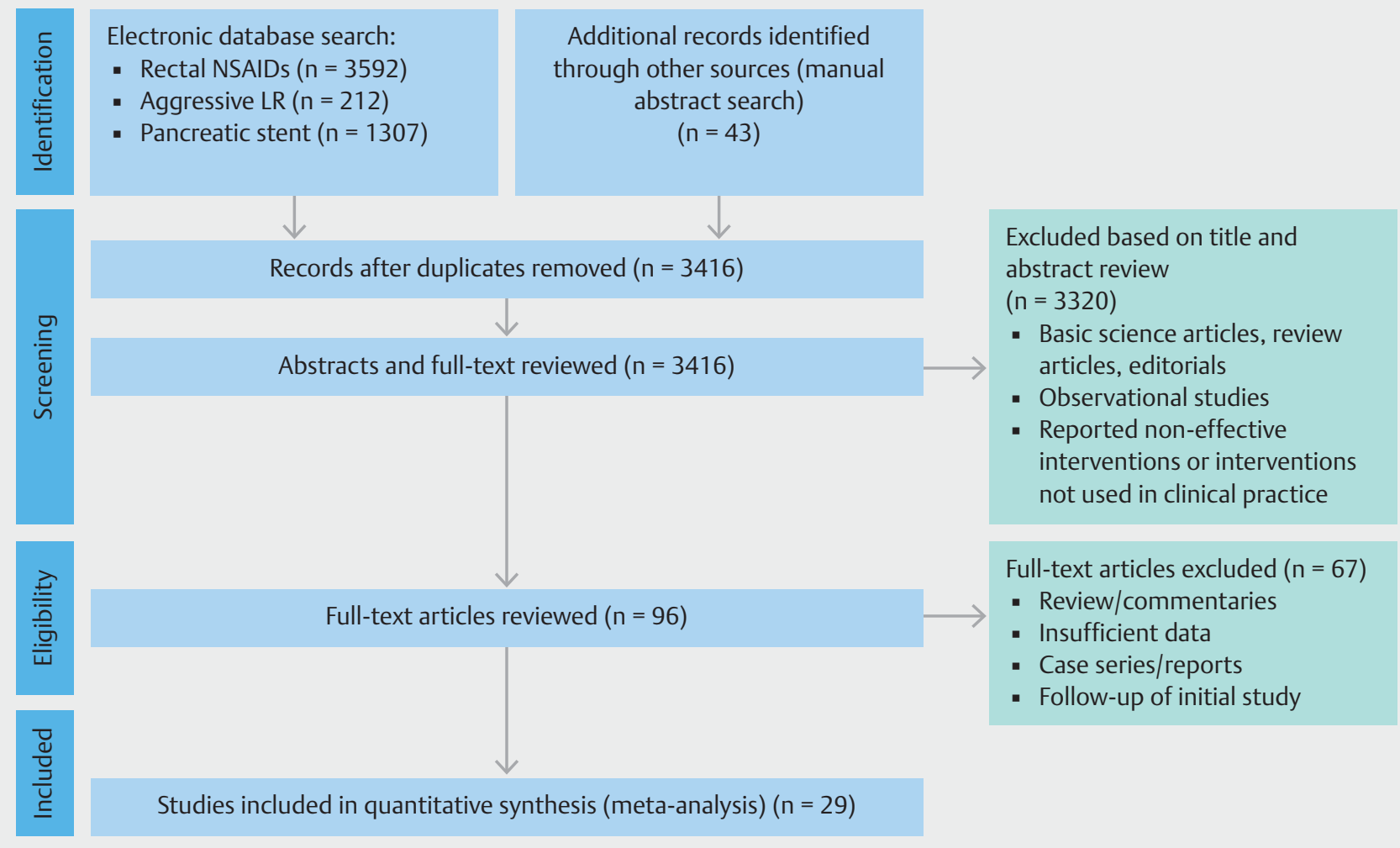

- Fig. 1 Preferred Reporting Items for Systematic Reviews and Meta-Analyses (PRISMA) flowchart of search results for the prevention of postERCP pancreatitis.

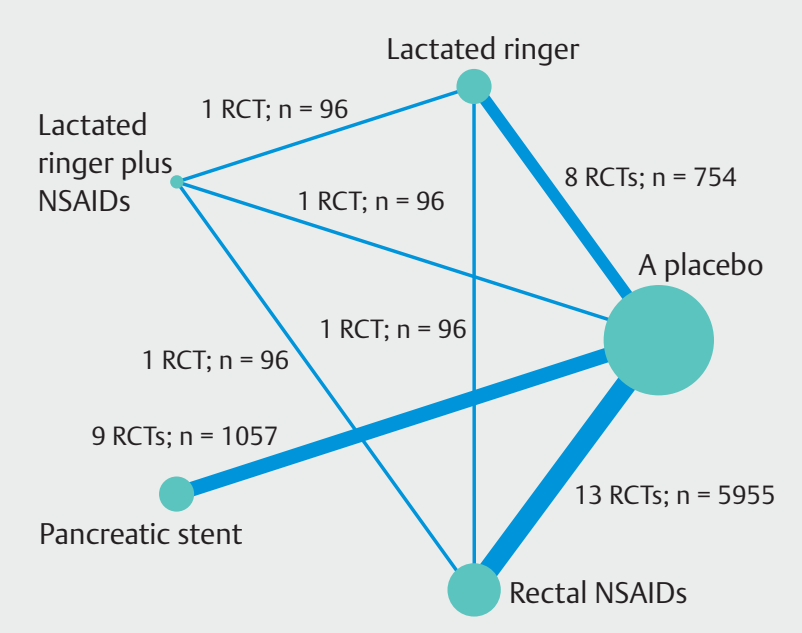

Fig. 2 Network meta-analysis design of included studies for the prevention of post-ERCP pancreatitis.

$75 \%$ reduced odds of developing post-ERCP pancreatitis; however, this was not statistically significant $(O R=0.25,95 \% \mathrm{Cl}$ [0.06 to 0.99]). Prophylactic pancreatic duct stent placement was effective in decreasing post-ERCP pancreatitis by $71 \%$ (OR $=0.29,95 \% \mathrm{Cl}[0.17$ to 0.48$]$ ) ( $\mathbf{F i g . 5}$ ).

\section{Sensitivity analyses}

Results from sensitivity analyses are reported in the Supplemental Table 1. Overall, for the primary outcome, the results were largely similar to the main analysis.

\section{Publication bias}

There was no evidence of publication bias, based upon either qualitative on funnel-plot asymmetry or quantitative (i.e., Egger regression test, $P>0.05$ for all comparisons) (Supplemental Fig. 3, Supplemental Fig. 4, Supplement Fig. 5).

\section{Network meta-analysis comparisons}

With this network meta-analysis, we calculated the mixed effect estimate as a weighted average of both direct and indirect treatment effects. On direct network meta-analysis, all strategies (i.e., both pharmacologic and endoscopic treatments) were associated with a reduced risk of post-ERCP pancreatitis. In a direct network meta-analysis, compared with placebo, use of rectal NSAIDs ( $\mathrm{B}=-0.69,95 \% \mathrm{Cl}[-1.18$ to -0.21$]$ ), pancreatic stent $(B=-1.25,95 \% \mathrm{Cl}[-1.81$ to -0.69$])$, high-volume LR solution $(B=-0.67,95 \% \mathrm{Cl}[-1.20$ to -0.13$])$, and combination of $L R$ plus rectal NSAIDs $(B=-1.58,95 \% \mathrm{Cl}[-3.0$ to $-0.17]$ ), were all associated with a reduced risk of post-ERCP pancreatitis. Summary results of our network meta-analysis are summarized in $\mathbf{~ T a b l e ~} 2$. Indirect comparisons were largely similar to those obtained in direct meta-analysis for treatment 
- Table 1 Summary characteristics of included studies for prevention of post-ERCP Pancreatitis.

\begin{tabular}{|c|c|c|c|c|c|c|c|c|c|}
\hline Author & $\begin{array}{l}\text { Year, } \\
\text { place } \\
\text { of } \\
\text { study }\end{array}$ & $\begin{array}{l}\text { Place } \\
\text { of } \\
\text { study }\end{array}$ & $\begin{array}{l}\text { Type of } \\
\text { manu- } \\
\text { script }\end{array}$ & Study design & $\begin{array}{l}\text { Sam- } \\
\text { ple } \\
\text { size }\end{array}$ & $\begin{array}{l}\text { Mean age of } \\
\text { treatment } \\
\text { group } \\
\text { (years) }\end{array}$ & $\begin{array}{l}\text { Female } \\
\text { gender } \\
\text { (\%) }\end{array}$ & $\begin{array}{l}\text { Primary ERCP } \\
\text { indication of } \\
\text { bile duct stone } \\
\text { (\%) }\end{array}$ & $\begin{array}{l}\text { Trial-specific treat- } \\
\text { ment details }\end{array}$ \\
\hline \multicolumn{10}{|c|}{ Rectal NSAID Therapy } \\
\hline $\begin{array}{l}\text { Murray et } \\
\text { al. }\end{array}$ & 2003 & $\begin{array}{l}\text { Scot- } \\
\text { land }\end{array}$ & $\begin{array}{l}\text { Full } \\
\text { text }\end{array}$ & $\begin{array}{l}\text { Single-center } \\
\text { comparator to } \\
\text { placebo }\end{array}$ & 220 & 55 & $65 \%$ & $25.45 \%$ & $\begin{array}{l}\text { Diclofenac } 100 \mathrm{mg} \text { im- } \\
\text { mediately post-ERCP }\end{array}$ \\
\hline $\begin{array}{l}\text { Sotoudeh- } \\
\text { manesh et } \\
\text { al. }\end{array}$ & 2007 & Iran & $\begin{array}{l}\text { Full } \\
\text { text }\end{array}$ & $\begin{array}{l}\text { Single-center } \\
\text { comparator to } \\
\text { placebo }\end{array}$ & 490 & 58.4 & $53.90 \%$ & $53.26 \%$ & $\begin{array}{l}\text { Indomethacin } 100 \mathrm{mg} \\
\text { immediately pre-ERCP }\end{array}$ \\
\hline $\begin{array}{l}\text { Otsuka et } \\
\text { al. }\end{array}$ & 2012 & Japan & $\begin{array}{l}\text { Full } \\
\text { text }\end{array}$ & $\begin{array}{l}\text { Multicenter } \\
\text { comparator to } \\
\text { placebo }\end{array}$ & 104 & 75 & $49.04 \%$ & $77.88 \%$ & $\begin{array}{l}\text { Diclofenac } 25-50 \mathrm{mg} \\
\text { immediately pre-ERCP }\end{array}$ \\
\hline $\begin{array}{l}\text { Elmunzer } \\
\text { et al. }\end{array}$ & 2012 & $\begin{array}{l}\text { United } \\
\text { States }\end{array}$ & $\begin{array}{l}\text { Full } \\
\text { text }\end{array}$ & $\begin{array}{l}\text { Multicenter } \\
\text { comparator to } \\
\text { placebo }\end{array}$ & 602 & 44.4 & $79.07 \%$ & - & $\begin{array}{l}\text { Indomethacin } 100 \mathrm{mg} \\
\text { immediately post- } \\
\text { ERCP }\end{array}$ \\
\hline $\begin{array}{l}\text { Dobronte } \\
\text { et al. }\end{array}$ & 2012 & $\begin{array}{l}\text { Hun- } \\
\text { gary }\end{array}$ & $\begin{array}{l}\text { Full } \\
\text { text }\end{array}$ & $\begin{array}{l}\text { Single-center } \\
\text { comparator to } \\
\text { placebo }\end{array}$ & 228 & - & - & - & $\begin{array}{l}\text { Indomethacin } 100 \mathrm{mg} \\
10-15 \text { minutes pre- } \\
\text { ERCP }\end{array}$ \\
\hline $\begin{array}{l}\text { Alabd and } \\
\text { Abo }\end{array}$ & 2013 & Sudan & $\begin{array}{l}\text { Ab- } \\
\text { stract }\end{array}$ & $\begin{array}{l}\text { Single-center } \\
\text { comparator to } \\
\text { placebo }\end{array}$ & 240 & - & - & - & - \\
\hline $\begin{array}{l}\text { Dobronte } \\
\text { et al. }\end{array}$ & 2014 & $\begin{array}{l}\text { Hun- } \\
\text { gary }\end{array}$ & $\begin{array}{l}\text { Full } \\
\text { text }\end{array}$ & $\begin{array}{l}\text { Single-center } \\
\text { comparator to } \\
\text { placebo }\end{array}$ & 665 & 66.8 & - & - & $\begin{array}{l}\text { Indomethacin } 100 \mathrm{mg} \\
10-15 \text { minutes pre- } \\
\text { ERCP }\end{array}$ \\
\hline $\begin{array}{l}\text { Andrade- } \\
\text { Davila et al. }\end{array}$ & 2015 & Mexico & $\begin{array}{l}\text { Full } \\
\text { text }\end{array}$ & $\begin{array}{l}\text { Single-center } \\
\text { comparator to } \\
\text { placebo }\end{array}$ & 166 & 51.59 & $66.27 \%$ & $39.76 \%$ & $\begin{array}{l}\text { Indomethacin } 100 \mathrm{mg} \\
\text { immediately post- } \\
\text { ERCP }\end{array}$ \\
\hline Patai et al. & 2015 & $\begin{array}{l}\text { Hun- } \\
\text { gary }\end{array}$ & $\begin{array}{l}\text { Full } \\
\text { text }\end{array}$ & $\begin{array}{l}\text { Single-center } \\
\text { comparator to } \\
\text { placebo }\end{array}$ & 574 & 66.25 & $67.16 \%$ & $58.63 \%$ & $\begin{array}{l}\text { Indomethacin } 100 \mathrm{mg} \\
60 \text { minutes pre-ERCP }\end{array}$ \\
\hline Lua et al. & 2015 & $\begin{array}{l}\text { Malay- } \\
\text { sia }\end{array}$ & $\begin{array}{l}\text { Full } \\
\text { text }\end{array}$ & $\begin{array}{l}\text { Single-center } \\
\text { comparator to } \\
\text { placebo }\end{array}$ & 151 & 50.3 & $59.03 \%$ & $56.25 \%$ & $\begin{array}{l}\text { Diclofenac } 100 \mathrm{mg} \mathrm{im-} \\
\text { mediately post-ERCP }\end{array}$ \\
\hline Luo et al. & 2016 & China & $\begin{array}{l}\text { Full } \\
\text { text }\end{array}$ & $\begin{array}{l}\text { Multicenter } \\
\text { comparator to } \\
\text { placebo }\end{array}$ & 2014 & 62 & $52.42 \%$ & $77.50 \%$ & $\begin{array}{l}\text { Indomethacin } 100 \mathrm{mg} \\
30 \text { minutes pre-ERCP }\end{array}$ \\
\hline $\begin{array}{l}\text { Levenick et } \\
\text { al. }\end{array}$ & 2016 & $\begin{array}{l}\text { United } \\
\text { States }\end{array}$ & $\begin{array}{l}\text { Full } \\
\text { text }\end{array}$ & $\begin{array}{l}\text { Single-center } \\
\text { comparator to } \\
\text { placebo }\end{array}$ & 449 & 64.9 & $52.56 \%$ & $27.72 \%$ & $\begin{array}{l}\text { Indomethacin } 50 \mathrm{mg} \times \\
2 \text { during ERCP }\end{array}$ \\
\hline Ucar et al. & 2016 & Turkey & $\begin{array}{l}\text { Full } \\
\text { text }\end{array}$ & $\begin{array}{l}\text { Single-center } \\
\text { comparator to } \\
\text { placebo }\end{array}$ & 100 & 59 & $66.66 \%$ & $83 \%$ & $\begin{array}{l}\text { Diclofenac } 100 \mathrm{mg} \\
30-90 \text { minutes pre- } \\
\text { ERCP }\end{array}$ \\
\hline \multicolumn{10}{|c|}{ Aggressive LR solution } \\
\hline $\begin{array}{l}\text { Buxbaum } \\
\text { et al. }\end{array}$ & 2014 & $\begin{array}{l}\text { United } \\
\text { States }\end{array}$ & $\begin{array}{l}\text { Full } \\
\text { text }\end{array}$ & $\begin{array}{l}\text { Single-center } \\
\text { comparator to } \\
\text { standard hy- } \\
\text { dration }\end{array}$ & 62 & 43 & $51.61 \%$ & $74.20 \%$ & $\begin{array}{l}\text { IV LR solution at a rate } \\
\text { of } 3.0 \mathrm{~mL} / \mathrm{kg} / \mathrm{h} \text { during } \\
\text { ERCP, a bolus of } 20 \mathrm{~mL} / \\
\mathrm{kg} \text { immediately post- } \\
\text { ERCP, followed by } \\
\text { post-ERCP rate of } \\
3.0 \mathrm{~mL} / \mathrm{kg} / \mathrm{h} \text { for } 8 \mathrm{~h}\end{array}$ \\
\hline
\end{tabular}


- Table 1 (Continuation)

\begin{tabular}{|c|c|c|c|c|c|c|c|c|c|}
\hline Author & $\begin{array}{l}\text { Year, } \\
\text { place } \\
\text { of } \\
\text { study }\end{array}$ & $\begin{array}{l}\text { Place } \\
\text { of } \\
\text { study }\end{array}$ & $\begin{array}{l}\text { Type of } \\
\text { manu- } \\
\text { script }\end{array}$ & Study design & $\begin{array}{l}\text { Sam- } \\
\text { ple } \\
\text { size }\end{array}$ & $\begin{array}{l}\text { Mean age of } \\
\text { treatment } \\
\text { group } \\
\text { (years) }\end{array}$ & $\begin{array}{l}\text { Female } \\
\text { gender } \\
\text { (\%) }\end{array}$ & $\begin{array}{l}\text { Primary ERCP } \\
\text { indication of } \\
\text { bile duct stone } \\
\text { (\%) }\end{array}$ & $\begin{array}{l}\text { Trial-specific treat- } \\
\text { ment details }\end{array}$ \\
\hline $\begin{array}{l}\text { Chuank- } \\
\text { rekkul et } \\
\text { al. }\end{array}$ & 2015 & $\begin{array}{l}\text { Thai- } \\
\text { land }\end{array}$ & $\begin{array}{l}\text { Ab- } \\
\text { stract }\end{array}$ & $\begin{array}{l}\text { Single-center } \\
\text { comparator to } \\
\text { standard hy- } \\
\text { dration }\end{array}$ & 60 & 61.9 & - & - & $\begin{array}{l}\text { IV LR solution at a rate } \\
\text { of } 3.0 \mathrm{~mL} / \mathrm{kg} / \mathrm{h} \text { during } \\
\text { ERCP, a bolus of } 10 \mathrm{~mL} / \\
\mathrm{kg} \text { immediately post- } \\
\text { ERCP, followed by } \\
\text { post-ERCP rate of } \\
3.0 \mathrm{~mL} / \mathrm{kg} / \mathrm{h} \text { for } 8 \mathrm{~h}\end{array}$ \\
\hline $\begin{array}{l}\text { Shaygan- } \\
\text { Nejad et al. }\end{array}$ & 2015 & Iran & $\begin{array}{l}\text { Full } \\
\text { text }\end{array}$ & $\begin{array}{l}\text { Single-center } \\
\text { comparator to } \\
\text { standard hy- } \\
\text { dration }\end{array}$ & 150 & 49.6 & $66 \%$ & $95.35 \%$ & $\begin{array}{l}\text { IV LR solution at a rate } \\
\text { of } 3.0 \mathrm{~mL} / \mathrm{kg} / \mathrm{h} \text { during } \\
\text { ERCP, a bolus of } 20 \mathrm{~mL} / \\
\mathrm{kg} \text { immediately post- } \\
\text { ERCP, followed by } \\
\text { post-ERCP rate of } \\
3.0 \mathrm{~mL} / \mathrm{kg} / \mathrm{h} \text { for } 8 \mathrm{~h}\end{array}$ \\
\hline Rosa et al. & 2016 & $\begin{array}{l}\text { Portu- } \\
\text { gal }\end{array}$ & $\begin{array}{l}\text { Ab- } \\
\text { stract }\end{array}$ & $\begin{array}{l}\text { Multicenter } \\
\text { comparator to } \\
\text { standard hy- } \\
\text { dration }\end{array}$ & 68 & - & - & - & $\begin{array}{l}\text { IV LR solution at a rate } \\
\text { of } 3.0 \mathrm{~mL} / \mathrm{kg} / \mathrm{h} \text { during } \\
\text { ERCP, a bolus of } 20 \mathrm{~mL} / \\
\mathrm{kg} \text { immediately post- } \\
\text { ERCP, followed by } \\
\text { post-ERCP rate of } \\
3.0 \mathrm{~mL} / \mathrm{kg} / \mathrm{h} \text { for } 8 \mathrm{~h}\end{array}$ \\
\hline $\begin{array}{l}\text { NC- } \\
\text { T0205004- } \\
8\end{array}$ & 2016 & $\begin{array}{l}\text { United } \\
\text { States }\end{array}$ & - & $\begin{array}{l}\text { Multicenter } \\
\text { comparator to } \\
\text { standard hy- } \\
\text { dration }\end{array}$ & 26 & 59.1 & $84.62 \%$ & - & $\begin{array}{l}\text { Initial bolus LR solu- } \\
\text { tion of } 7.58 \mathrm{~mL} / \mathrm{kg} \text { pre- } \\
\text { ERCP, IV LR solution of } \\
5.0 \mathrm{~mL} / \mathrm{kg} / \mathrm{h} \text { during } \\
\text { ERCP, following by } \\
\text { post-ERCP bolus of } \\
20 \mathrm{~mL} / \mathrm{kg} \text { for } 90 \mathrm{~min} \text { - } \\
\text { utes }\end{array}$ \\
\hline $\begin{array}{l}\text { Chang et } \\
\text { al. }\end{array}$ & 2016 & $\begin{array}{l}\text { Thai- } \\
\text { land }\end{array}$ & $\begin{array}{l}\text { Ab- } \\
\text { stract }\end{array}$ & $\begin{array}{l}\text { Single-center } \\
\text { comparator to } \\
\text { standard hy- } \\
\text { dration }\end{array}$ & 171 & - & - & $50 \%$ & $\begin{array}{l}\text { IV LR solution at a rate } \\
\text { of } 150 \mathrm{~mL} / \mathrm{h} \text { starting } \\
2 \mathrm{~h} \text { pre-ERCP, and con- } \\
\text { tinued during and } \\
\text { post-ERCP for } 24 \mathrm{~h}\end{array}$ \\
\hline Choi et al. & 2016 & Korea & $\begin{array}{l}\text { Full } \\
\text { text }\end{array}$ & $\begin{array}{l}\text { Multicenter } \\
\text { comparator to } \\
\text { standard hy- } \\
\text { dration }\end{array}$ & 510 & 57.6 & $45.50 \%$ & $53.70 \%$ & $\begin{array}{l}\text { Initial bolus } L R \text { solu- } \\
\text { tion of } 10 \mathrm{~mL} / \mathrm{kg} \text { pre- } \\
\text { ERCP, IV LR solution of } \\
3.0 \mathrm{~mL} / \mathrm{kg} / \mathrm{h} \text { during } \\
\text { ERCP and continued } \\
8 \mathrm{~h} \text { post-ERCP, follow- } \\
\text { ing by post-ERCP bolus } \\
\text { of } 10 \mathrm{~mL} / \mathrm{kg}\end{array}$ \\
\hline Mok et al. & 2017 & $\begin{array}{l}\text { United } \\
\text { States }\end{array}$ & $\begin{array}{l}\text { Full } \\
\text { text }\end{array}$ & $\begin{array}{l}\text { Single-center } \\
\text { comparator } \\
\text { with multiple } \\
\text { therapies* }\end{array}$ & 48 & 60.25 & $60.60 \%$ & - & $\begin{array}{l}\text { Treatment arms in- } \\
\text { cluded standard nor- } \\
\text { mal saline solution vs } \\
\text { normal saline plus in- } \\
\text { domethacin versus LR } \\
\text { versus LR plus indo- } \\
\text { methacin }\end{array}$ \\
\hline
\end{tabular}


- Table 1 (Continuation)

\begin{tabular}{|c|c|c|c|c|c|c|c|c|c|}
\hline Author & $\begin{array}{l}\text { Year, } \\
\text { place } \\
\text { of } \\
\text { study }\end{array}$ & $\begin{array}{l}\text { Place } \\
\text { of } \\
\text { study }\end{array}$ & $\begin{array}{l}\text { Type of } \\
\text { manu- } \\
\text { script }\end{array}$ & Study design & $\begin{array}{l}\text { Sam- } \\
\text { ple } \\
\text { size }\end{array}$ & $\begin{array}{l}\text { Mean age of } \\
\text { treatment } \\
\text { group } \\
\text { (years) }\end{array}$ & $\begin{array}{l}\text { Female } \\
\text { gender } \\
\text { (\%) }\end{array}$ & $\begin{array}{l}\text { Primary ERCP } \\
\text { indication of } \\
\text { bile duct stone } \\
\text { (\%) }\end{array}$ & $\begin{array}{l}\text { Trial-specific treat- } \\
\text { ment details }\end{array}$ \\
\hline \multicolumn{10}{|c|}{ Rectal NSAIDs + LR solution } \\
\hline Mok et al. & 2017 & $\begin{array}{l}\text { United } \\
\text { States }\end{array}$ & $\begin{array}{l}\text { Full } \\
\text { text }\end{array}$ & $\begin{array}{l}\text { Single-center } \\
\text { comparator } \\
\text { with multiple } \\
\text { therapies* }\end{array}$ & 48 & 60.25 & $60.60 \%$ & - & $\begin{array}{l}\text { Treatment arms in- } \\
\text { cluded standard nor- } \\
\text { mal saline solution vs } \\
\text { normal saline plus in- } \\
\text { domethacin versus LR } \\
\text { versus LR plus indo- } \\
\text { methacin }\end{array}$ \\
\hline \multicolumn{10}{|c|}{ Pancreatic stent placement } \\
\hline $\begin{array}{l}\text { Smithline } \\
\text { et al. }\end{array}$ & 1993 & $\begin{array}{l}\text { United } \\
\text { States }\end{array}$ & $\begin{array}{l}\text { Full } \\
\text { text }\end{array}$ & $\begin{array}{l}\text { Single-center } \\
\text { comparator to } \\
\text { placebo }\end{array}$ & 99 & 46 & $78.79 \%$ & - & $\begin{array}{l}5-7 \mathrm{Fr} \text { stent, } \\
2-2.5 \mathrm{~cm} \text { in length }\end{array}$ \\
\hline $\begin{array}{l}\text { Tarnasky et } \\
\text { al. }\end{array}$ & 1998 & $\begin{array}{l}\text { United } \\
\text { States }\end{array}$ & $\begin{array}{l}\text { Full } \\
\text { text }\end{array}$ & $\begin{array}{l}\text { Single-center } \\
\text { comparator to } \\
\text { placebo }\end{array}$ & 80 & 46.05 & - & - & $\begin{array}{l}5-7 \mathrm{Fr} \text { stent, } \\
2-2.5 \mathrm{~cm} \text { in length }\end{array}$ \\
\hline Fazel et al. & 2003 & $\begin{array}{l}\text { United } \\
\text { States }\end{array}$ & $\begin{array}{l}\text { Full } \\
\text { text }\end{array}$ & $\begin{array}{l}\text { Single-center } \\
\text { comparator to } \\
\text { placebo }\end{array}$ & 74 & 44.7 & $86.49 \%$ & - & $\begin{array}{l}\text { Nasopancreatic } 5 \mathrm{Fr} \\
\text { catheter or } 5 \mathrm{Fr} \text { stent, } \\
2 \mathrm{~cm} \text { length, } 2 \text { barbed }\end{array}$ \\
\hline $\begin{array}{l}\text { Harewood } \\
\text { et al. }\end{array}$ & 2005 & $\begin{array}{l}\text { United } \\
\text { States }\end{array}$ & $\begin{array}{l}\text { Full } \\
\text { text }\end{array}$ & $\begin{array}{l}\text { Single-center } \\
\text { comparator to } \\
\text { placebo }\end{array}$ & 19 & 48.75 & $63.16 \%$ & - & $\begin{array}{l}\text { Straight, single flan- } \\
\text { ged, polyethylene } 5 \mathrm{Fr} \\
\text { stent, } 3-5 \mathrm{~cm} \text { length }\end{array}$ \\
\hline $\begin{array}{l}\text { Sofuni et } \\
\text { al. }\end{array}$ & 2011 & Japan & $\begin{array}{l}\text { Full } \\
\text { text }\end{array}$ & $\begin{array}{l}\text { Multicenter } \\
\text { comparator to } \\
\text { placebo }\end{array}$ & 201 & 66.4 & $37.81 \%$ & - & $\begin{array}{l}\text { Straight, } 5 \mathrm{Fr} \text { stent, } \\
3 \mathrm{~cm} \text { in length }\end{array}$ \\
\hline Pan et al. & 2012 & China & $\begin{array}{l}\text { Full } \\
\text { text }\end{array}$ & $\begin{array}{l}\text { Single-center } \\
\text { comparator to } \\
\text { placebo }\end{array}$ & 40 & 58.3 & $52.50 \%$ & - & $\begin{array}{l}\text { Single pigtail, } 5 \mathrm{Fr} \\
\text { stent }\end{array}$ \\
\hline Lee et al. & 2012 & Korea & $\begin{array}{l}\text { Full } \\
\text { text }\end{array}$ & $\begin{array}{l}\text { Single-center } \\
\text { comparator to } \\
\text { placebo }\end{array}$ & 101 & 57.5 & $62.38 \%$ & $66.33 \%$ & $\begin{array}{l}\text { Single pigtail unflan- } \\
\text { ged } 3 \mathrm{Fr} \text { stent, } 4-8 \mathrm{~cm} \\
\text { length }\end{array}$ \\
\hline $\begin{array}{l}\text { Kawaguchi } \\
\text { et al. }\end{array}$ & 2012 & Japan & $\begin{array}{l}\text { Full } \\
\text { text }\end{array}$ & $\begin{array}{l}\text { Single-center } \\
\text { comparator to } \\
\text { placebo }\end{array}$ & 120 & 67 & $56.67 \%$ & $35.83 \%$ & $\begin{array}{l}\text { Unflanged on pancre- } \\
\text { atic duct side, } 2 \text { flan- } \\
\text { ges on duodenal side } \\
5 \text { Fr stent, } 3 \mathrm{~cm} \text { length }\end{array}$ \\
\hline Cha et al. & 2013 & $\begin{array}{l}\text { United } \\
\text { States }\end{array}$ & $\begin{array}{l}\text { Full } \\
\text { text }\end{array}$ & $\begin{array}{l}\text { Single-center } \\
\text { comparator to } \\
\text { placebo }\end{array}$ & 151 & 56.6 & $58.94 \%$ & $15.89 \%$ & $\begin{array}{l}\text { Straight or external } \\
3 / 4 \text { pigtail } 5-7 \mathrm{Fr} \\
\text { stent, } 2-2.5 \mathrm{~cm} \\
\text { length }\end{array}$ \\
\hline
\end{tabular}

ERCP, endoscopic retrograde cholangiopancreatography; NSAID, nonsteroidal anti-inflammatory drug; IV, intravenous; LR, lactated ringer's

modalities compared to placebo. However, in indirect network meta-analysis, there was no statistically significant difference when treatment modalities were compared with each other. There were overlapping confidence intervals, although differences were observed in effect size.

\section{Ranking probability}

Ranking probability based on SUCRA indicated that prophylactic pancreatic duct stent placement had the highest probability (SUCRA $=0.81,95 \% \mathrm{Cl}[0.83$ to 0.80$]$ ) of being ranked the best prophylactic treatment ( $\nabla \mathbf{F i g . 6}$ ). As highlighted in the SUCRA probabilities figure, a combined strategy of LR solution plus rectal NSAIDs was the second most effective treatment to prevent post-ERCP pancreatitis (SUCRA $=0.76,95 \% \mathrm{Cl}[0.79$ to $0.72]$ ), followed by aggressive infusion of LR solution alone ( $\mathrm{SU}$ - 


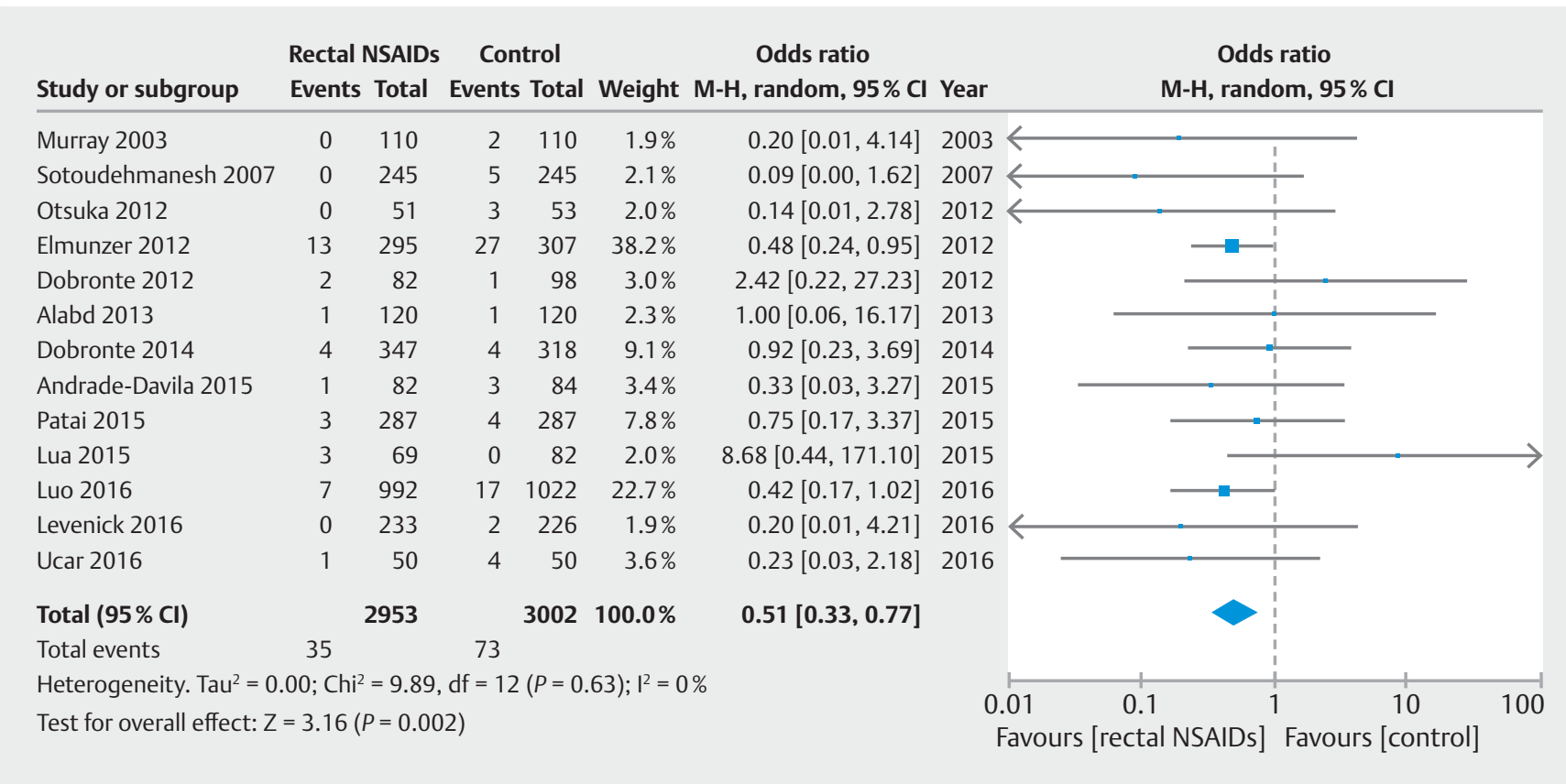

- Fig. 3 Direct treatment comparison of rectal NSAIDs to placebo for the prevention of post-ERCP pancreatitis.

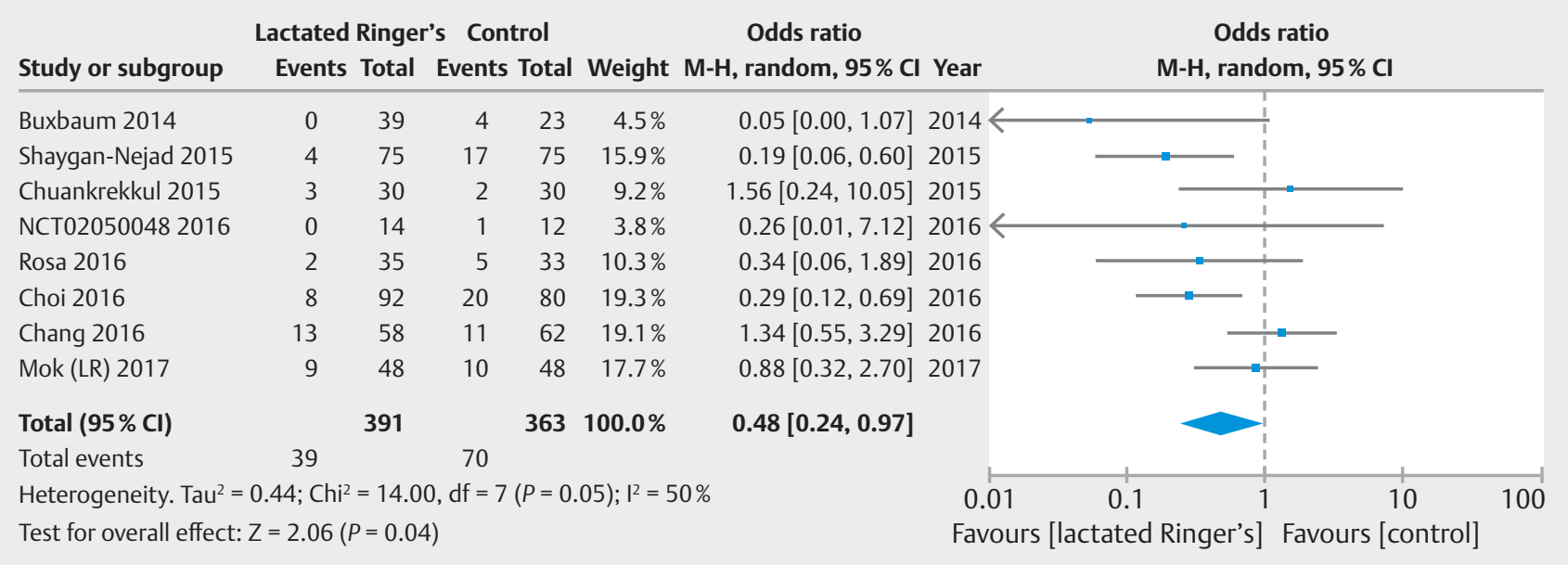

Fig.4 Direct treatment comparison of aggressive LR solution to placebo for the prevention of post-ERCP pancreatitis.

$\mathrm{CRA}=0.51,95 \% \mathrm{Cl}[0.55$ to 0.49$])$, and finally independent use of rectal NSAID use (SUCRA $=0.41,95 \% \mathrm{Cl}[0.44$ to 0.38$]$ ). A rankogram of interventions to prevent post-ERCP pancreatitis based upon cumulative SUCRA probabilities is highlighted in Supplemental Fig. 6.

\section{Discussion}

In this systematic review and network meta-analysis, compared to other treatment approaches, pancreatic duct stent placement appears to be the most effective strategy for the prevention of post-ERCP pancreatitis followed by prophylaxis using aggressive LR hydration and rectal NSAIDs, followed by high-volume LR infusions alone, and finally use of rectal NSAIDs.
Pancreatic duct stents reduce post-ERCP pancreatitis by relieving pancreatic ductal hypertension that may develop as a result of transient procedure-induced stenosis [58]. The most recent European Society of Gastrointestinal Endoscopy (ESGE) guideline on prophylaxis of post-ERCP pancreatitis recommends placement of a $5 \mathrm{Fr}$ prophylactic pancreatic stent in high-risk cases [59]. However, pancreatic stent placement is technically challenging. Pancreatic stent requires deep cannulation of the duct and placement of a guidewire. Attempting to place a pancreatic duct stent with subsequent failure actually increases risk of post-ERCP pancreatitis by inducing injury to the pancreatic orifice. Hence, it is these authors' belief that pancreatic stent placement should only be attempted by providers with familiarity and expertise, in individuals deemed to be 


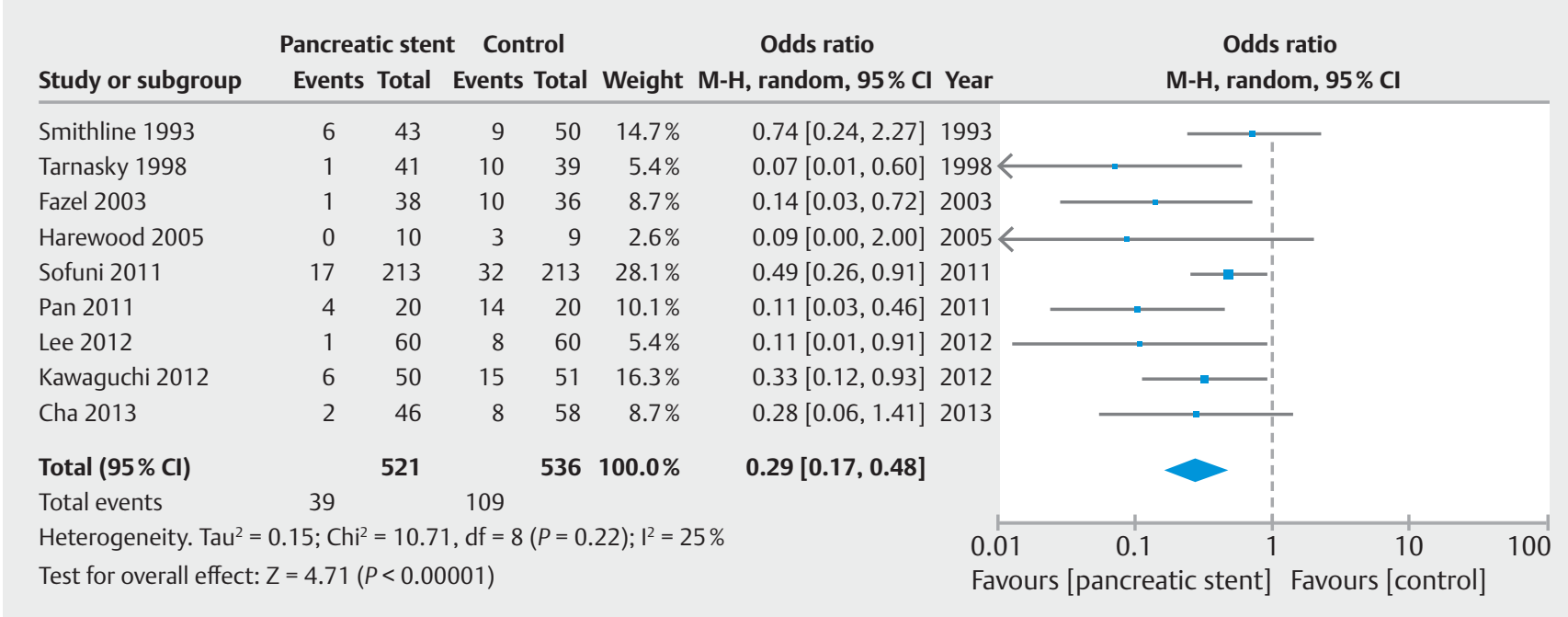

- Fig. 5 Direct treatment comparison of pancreatic stent placement to placebo for the prevention of post-ERCP pancreatitis.

- Table 2 Summary results from network meta-analysis in the prevention of post-ERCP pancreatitis.

\begin{tabular}{|c|c|c|c|}
\hline Post-ERCP treatment & Beta coefficient $(\beta)$ & $95 \%$ credible interval $(\mathrm{CI})^{1}$ & Quality of evidence \\
\hline Lactated ringer's vs placebo & -0.69 & -0.77 to -2.15 & $\begin{array}{l}\oplus \oplus \oplus \\
\text { Moderate }^{2}\end{array}$ \\
\hline Lactated ringer's + rectal nsaids vs placebo & -0.82 & -1.45 to -0.19 & $\begin{array}{l}\oplus \oplus \oplus \oplus \\
\text { High }\end{array}$ \\
\hline Pancreatic stent vs placebo & -1.37 & -2.98 to -0.23 & $\begin{array}{l}\oplus \oplus \oplus \oplus \\
\text { High }\end{array}$ \\
\hline Rectal NSAIDs vs placebo & -1.27 & -2.30 to -0.70 & $\begin{array}{l}\oplus \oplus \oplus \oplus \\
\text { High }\end{array}$ \\
\hline Lactated ringer's + rectal NSAIDs vs lactated ringer's & -0.55 & -2.28 to 1.17 & $\begin{array}{l}\oplus \oplus \oplus \\
\text { Low }^{3}\end{array}$ \\
\hline Pancreatic stent vs lactated ringer's & -0.45 & -1.30 to 0.39 & $\begin{array}{l}\oplus \oplus \oplus \\
\text { Low }^{3}\end{array}$ \\
\hline Rectal NSAIDs vs lactated ringer's & 0.21 & -1.32 to 1.74 & $\begin{array}{l}\oplus \oplus \oplus \\
\text { Low }^{3}\end{array}$ \\
\hline Pancreatic stent vs lactated ringer's + rectal NSAIDs & 0.10 & -1.61 to 1.80 & $\begin{array}{l}\oplus \oplus \oplus \\
\text { Low }^{3}\end{array}$ \\
\hline Rectal NSAIDs vs lactated ringer's + rectal NSAIDs & 0.76 & -0.92 to 2.44 & $\begin{array}{l}\oplus \oplus \oplus \\
\text { Low }^{3}\end{array}$ \\
\hline Rectal NSAIDs vs pancreatic stent & 0.66 & -0.84 to 2.17 & $\begin{array}{l}\oplus \oplus \oplus \\
\text { Low }^{3}\end{array}$ \\
\hline \multicolumn{4}{|c|}{$\begin{array}{l}\text { ERCP, endoscopic retrograde cholangiopancreatography; NSAID, nonsteroidal anti-inflammatory drug } \\
1 \text { If the Cl estimates are either all positive or negative (i. e., does not include a zero), it indicates that results are statistically significant. } \\
2 \text { Due to risk of bias in imprecision of summary results. } \\
{ }^{3} \text { Due to risk of bias in individual studies, indirectness, and imprecision of summary results. }\end{array}$} \\
\hline
\end{tabular}

a high-risk, and in cases in which cannulation is not intentional and stent placement would be recommended in case of inadvertent cannulation. In patients in whom pancreatic stent placement is not feasible, use of alternative measures to reduce post-procedure pancreatitis should be employed.

Based on our study, aggressive hydration with LR appears to be effective when combined with rectal NSAIDs rather than using rectal NSAIDs alone or LR alone. Use of rectal NSAIDs, potent inhibitors of phospholipase $\mathrm{A} 2$, has been increasingly adopted in to clinical practice $[59,60]$. Current ESGE guidelines recommend routine rectal administration of diclofenac or indomethacin immediately before or after ERCP in all patients without contraindication [59]. Aggressive hydration with LR, which attenuates the acidosis that appears to promote zymogen acti- 


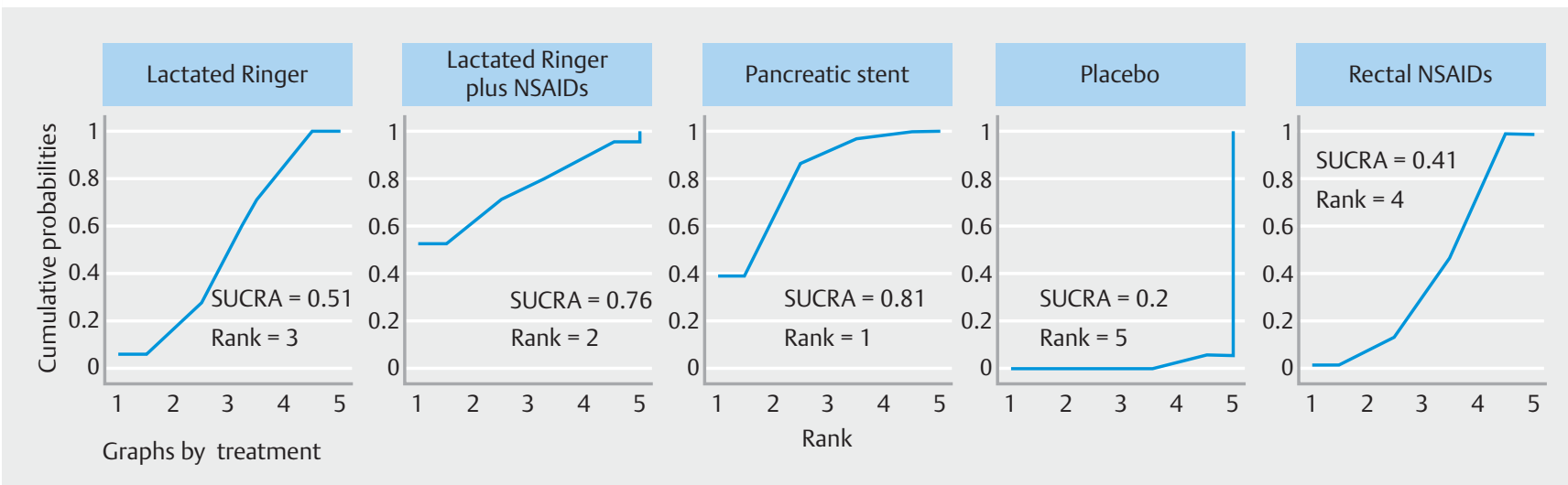

- Fig. 6 SUCRA cumulative probability plots for the prevention of post-ERCP pancreatitis.

vation and pancreatic inflammation, may be an effective intervention for preventing post-ERCP pancreatitis. Given the different mechanisms to prevent pancreatitis, it is not surprising that both work synergistically. Moreover, as both rectal NSAID therapy and LR infusion are inexpensive with relative few AEs, combination strategy is now has become a preferred strategy for post-ERCP pancreatitis prevention.

Our study observations are different from a previous network meta-analysis which reported that use of rectal NSAIDs when indirectly compared with pancreatic stent placement alone were associated with lower odds for developing postERCP pancreatitis [61]. However, that analysis included both observational studies and randomized controlled trials, which may have contributed to the divergent results. Furthermore, this previous study examined the efficacy of rectal NSAIDs and prophylactic pancreatic duct stent placement among both average-risk and high-risk cohorts. Our study specifically included only high-quality randomized controlled trials in highrisk patients and demonstrates the superiority of pancreatic stent placement as the best prophylactic measure for postERCP pancreatitis. Importantly, these findings do not take into account the cost-effectiveness of various therapies (i. e., rectal NSAIDs are inexpensive, easy to use, efficacious, and low risk). Despite our findings, questions still remain about whether all high-risk patients should receive a pancreatic stent-especially individuals who require multiple cannulation attempts of the biliary orifice.

It is important to note that the current systematic review and network meta-analysis is not without limitations. There were no studies comparing treatment strategies other than placebo except for one study that compared aggressive LR hydration to use of rectal NSAIDs. No currently published studies have examined the role of pancreatic duct stent versus rectal NSAIDs; although it should be pointed out there is an actively recruiting clinical trial designed to directly compare these two treatment modalities [62]. This study, with an expected enrollment of over 1400 patients, is a comparative effectiveness multicenter, non-inferiority trial of rectal indomethacin alone versus the combination of rectal indomethacin and prophylactic pancreatic stent placement for prevention of post-ERCP pancreatitis.

While we included indirect comparison in a Bayesian network meta-analysis design, we cannot discount the possibility of conceptual heterogeneity whereby differences may exist in trial design, patient population, intervention (i.e., timing of rectal NSAID administration or operator variability in pancreatic duct stent placement), and outcome assessment which may limit true comparability between included studies [19,27]. Though we performed sensitivity analyses to minimize heterogeneity and generalize our findings, it is important to understand that not all risk factors for post-ERCP pancreatitis are created equal, and combinations or risk factors are not simply additive, but multiplicative [63-66]. Additional limitations include a lack of data on follow-up - though the primary endpoint of post-ERCP pancreatitis lends itself to definitive diagnosis as defined over a discrete period of time as detailed above [25]. Treatment-related AEs were not reported in our systematic review and network meta-analysis as well, which may limit clinical adoption of these strategies, especially pancreatic duct stent placement.

Despite these limitations, our study possesses several strengths. These include the comprehensive and simultaneous assessment of the relative efficacy of four competing or incombination strategies to prevent post-ERCP pancreatitis in a high-risk cohort. Although SUCRA probabilities may not easily substitute for clinical judgement on a patient-by-patient basis, focusing on summary effect estimates and GRADE to rate overall quality of evidence allows for better approximations [27]. In addition, while conceptual heterogeneity was present in our study, strategies to limit the effect of conceptual heterogeneity included strict inclusion and exclusion criteria and use of multiple sensitivity analyses to assess the robustness of the results.

Furthermore, although there are several available strategies available to potentially reduce incidence post-ERCP pancreatitis, our results validate the current American Society of Gastrointestinal Endoscopy (ASGE) guidelines recommending pancreatic duct stenting in high-risk individuals - strong recommendation [22]. Future trials such as the one discussed above to assess rectal indomethacin alone versus combination therapy 
with rectal NSAID and prophylactic pancreatic stent placement may help development of future recommendations; however, these are unlikely to occur [62]. Comparison of these different strategies alone may be of limited benefit clinically because the combination of the different treatments has already readily been adopted in clinical practice and shown to be effective with few complications.

\section{Conclusion}

In conclusion, based upon this systematic review and network meta-analysis, prophylactic pancreatic stent placement appears to be the most effective preventive strategy for postERCP pancreatitis in high-risk patients. Despite these findings, it is these authors' belief that combination therapy will likely predominate clinical practice. This is in part due to the increased risk of post-ERCP pancreatitis with failed cannulation of the pancreatic duct and the ease and cost of combination therapy with rectal NSAID use and aggressive LR infusion.

\section{Competing interests}

Dr. Shyam Varadarajulu is a consultant for Boston Scientific and Olympus. Dr. Udayakumar Navaneethan is a consultant for Janssen, AbbVie, Pfizer and Takeda.

\section{References}

[1] Peery AF, Dellon ES, Lund J et al. Burden of gastrointestinal disease in the United States: 2012 update. Gastroenterology 2012; 143: 11791187 e1-3

[2] Sherman S. ERCP and endoscopic sphincterotomy-induced pancreatitis. Am J Gastroenterol 1994; 89: 303-305

[3] Silviera ML, Seamon M], Porshinsky B et al. Complications related to endoscopic retrograde cholangiopancreatography: a comprehensive clinical review. J Gastrointestin Liver Dis 2009; 18: 73 - 82

[4] Freeman ML, Nelson DB, Sherman $S$ et al. Complications of endoscopic biliary sphincterotomy. N Engl J Med 1996; 335: 909-918

[5] Glomsaker T, Hoff G, Kvaloy JT et al. Patterns and predictive factors of complications after endoscopic retrograde cholangiopancreatography. Br J Surg 2013; 100: 373-380

[6] Kochar B, Akshintala VS, Afghani E et al. Incidence, severity, and mortality of post-ERCP pancreatitis: a systematic review by using randomized, controlled trials. Gastrointest Endosc 2015; 81: $143-$ 149 e9

[7] Arata S, Takada T, Hirata K et al. Post-ERCP pancreatitis. J Hepatobiliary Pancreat Sci 2010; 17: $70-78$

[8] Murray B, Carter R, Imrie C et al. Diclofenac reduces the incidence of acute pancreatitis after endoscopic retrograde cholangiopancreatography. Gastroenterology 2003; 124: 1786-1791

[9] Otsuka T, Kawazoe S, Nakashita S et al. Low-dose rectal diclofenac for prevention of post-endoscopic retrograde cholangiopancreatography pancreatitis: a randomized controlled trial. J Gastroenterol 2012; 47: 912-917

[10] Sotoudehmanesh R, Khatibian M, Kolahdoozan S et al. Indomethacin may reduce the incidence and severity of acute pancreatitis after ERCP. Am J Gastroenterol 2007; 102: 978-983
[11] Chang AS, Pausawasdi N, Charatcharoenwitthaya P et al. A randomized, controlled trial of aggressive fluid hydration for the prevention of post-ERCP pancreatitis. Gastroenterol 2016; 150: S209

[12] Buxbaum J, Yan A, Yeh K et al. Aggressive hydration with lactated Ringer's solution reduces pancreatitis after endoscopic retrograde cholangiopancreatography. Clin Gastroenterol Hepatol 2014; 12: $303-307$ e1

[13] Choi JH, Kim HJ, Lee BU et al. Vigorous periprocedural hydration with lactated ringer's solution reduces the risk of pancreatitis after retrograde cholangiopancreatography in hospitalized patients. Clin Gastroenterol Hepatol 2017; 15: 86-92 e1

[14] Fazel A, Quadri A, Catalano MF et al. Does a pancreatic duct stent prevent post-ERCP pancreatitis? A prospective randomized study Gastrointest Endosc 2003; 57: 291 - 294

[15] Smithline A, Silverman W, Rogers D et al. Effect of prophylactic main pancreatic duct stenting on the incidence of biliary endoscopic sphincterotomy-induced pancreatitis in high-risk patients. Gastrointest Endosc 1993; 39: 652 - 657

[16] Tarnasky PR, Palesch YY, Cunningham JT et al. Pancreatic stenting prevents pancreatitis after biliary sphincterotomy in patients with sphincter of Oddi dysfunction. Gastroenterology 1998; 115: 1518 1524

[17] Dobronte Z, Szepes Z, Izbeki F et al. Is rectal indomethacin effective in preventing of post-endoscopic retrograde cholangiopancreatography pancreatitis? World J Gastroenterol 2014; 20: 10151-10157

[18] Hutton B, Salanti G, Chaimani A et al. The quality of reporting methods and results in network meta-analyses: an overview of reviews and suggestions for improvement. PLoS One 2014; 9: e92508

[19] Cipriani A, Higgins JP, Geddes JR et al. Conceptual and technical challenges in network meta-analysis. Ann Intern Med 2013; 159: 130 137

[20] Mills EJ, loannidis JP, Thorlund K et al. How to use an article reporting a multiple treatment comparison meta-analysis. JAMA 2012; 308: $1246-1253$

[21] Lu G, Ades AE. Combination of direct and indirect evidence in mixed treatment comparisons. Stat Med 2004; 23: 3105-3124

[22] Chandrasekhara V, Khashab MA et al. ASGE Standards of Practice Committee. Adverse events associated with ERCP. Gastrointest Endosc 2017; 85: $32-47$

[23] Testoni PA, Mariani A, Giussani A et al. Risk factors for post-ERCP pancreatitis in high- and low-volume centers and among expert and non-expert operators: a prospective multicenter study. Am J Gastroenterol 2010; 105: 1753-1761

[24] Chen JJ, Wang XM, Liu XQ et al. Risk factors for post-ERCP pancreatitis: a systematic review of clinical trials with a large sample size in the past 10 years. Eur J Med Res 2014; 19: 26

[25] Cotton PB, Eisen GM, Aabakken L et al. A lexicon for endoscopic adverse events: report of an ASGE workshop. Gastrointest Endosc 2010; 71: $446-454$

[26] Higgins JPT, Altman DG, Sterne JAC. Cochrane Handbook for Systematic Reviews of Interventions. In: Higgins JPT, ed. The Cochrane Collaboration; 2011: Available from http://www.cochranehandbook. org

[27] Puhan MA, Schunemann H], Murad MH et al. A GRADE Working Group approach for rating the quality of treatment effect estimates from network meta-analysis. BMJ 2014; 349: g5630

[28] Guyatt G, Oxman AD, Sultan S et al. GRADE guidelines: 11. Making an overall rating of confidence in effect estimates for a single outcome and for all outcomes. J Clin Epidemiol 2013; 66: 151-157

[29] DerSimonian R, Laird N. Meta-analysis in clinical trials. Control Clin Trials 1986; 7: $177-188$

[30] White IR. Multivariate random-effects meta-regression: Updates to mvmeta. The Stata Journal 2011; 11: 255-270 
[31] Higgins JP, Thompson SG, Deeks J] et al. Measuring inconsistency in meta-analyses. BMJ 2003; 327: 557-560

[32] Egger M, Davey SmithG, Schneider M et al. Bias in meta-analysis detected by a simple, graphical test. BMJ 1997; 315: 629-634

[33] Review Manager (RevMan), ver. 5.3. The Cochrane Collaboration; (ed.) 2014

[34] Lu G, Ades AE. Combination of direct and indirect evidence in mixed treatment comparisons. Stat Med 2004; 23: 3105-3124

[35] Caldwell DM, Ades AE, Higgins JP. Simultaneous comparison of multiple treatments: combining direct and indirect evidence. BMJ 2005; 331: $897-900$

[36] Chaimani A, Higgins JP, Mavridis D et al. Graphical tools for network meta-analysis in STATA. PLoS One 2013; 8: e76654

[37] Salanti G, Ades AE, Ioannidis JP. Graphical methods and numerical summaries for presenting results from multiple-treatment meta-analysis: an overview and tutorial. J Clin Epidemiol 2011; 64: 163-171

[38] Dobronte Z, Toldy E, Mark L et al. [Effects of rectal indomethacin in the prevention of post-ERCP acute pancreatitis]. Orv Hetil 2012; 153 : $990-996$

[39] Andrade-Davila VF, Chavez-Tostado M, Davalos-Cobian C et al. Rectal indomethacin versus placebo to reduce the incidence of pancreatitis after endoscopic retrograde cholangiopancreatography: results of a controlled clinical trial. BMC Gastroenterol 2015; 15: 85

[40] Patai A, Solymosi N, Patai AV. Effect of rectal indomethacin for preventing post-ERCP pancreatitis depends on difficulties of cannulation: results from a randomized study with sequential biliary intubation. J Clin Gastroenterol 2015; 49: 429-437

[41] Lua GW, Muthukaruppan R, Menon J. Can rectal diclofenac prevent post endoscopic retrograde cholangiopancreatography pancreatitis? Dig Dis Sci 2015; 60: $3118-3123$

[42] Luo H, Zhao L, Leung J et al. Routine pre-procedural rectal indometacin versus selective post-procedural rectal indometacin to prevent pancreatitis in patients undergoing endoscopic retrograde cholangiopancreatography: a multicentre, single-blinded, randomised controlled trial. Lancet 2016; 387: 2293-2301

[43] Levenick JM, Gordon SR, Fadden LL et al. Rectal indomethacin does not prevent post-ERCP pancreatitis in consecutive patients. Gastroenterology 2016; 150: 911 - 917; quiz e19.

[44] Ucar R, Biyik M, Ucar E et al. Rectal or intramuscular diclofenac reduces the incidence of pancreatitis afterendoscopic retrograde cholangiopancreatography. Turk J Med Sci 2016; 46: 1059-1063

[45] Alabd M, Abdo A. Role of rectal NSAIDs in the prevention of post-ERCP pancreatitis. J Gastroenterol Hepatol 2013; 28: 495-496

[46] Elmunzer BJ, Scheiman JM, Lehman GA et al. A randomized trial of rectal indomethacin to prevent post-ERCP pancreatitis. N Engl J Med 2012; 366: $1414-1422$

[47] Chuankrerkkul P. Aggressive lactated Ringer's solution for prevention of post ERCP pancreatitis (preliminary data of a prospective randomized trial). Gastrointest Endosc 2015; 81: AB410-AB411

[48] Rosa B, Carvalho PB, De Castro FD et al. Impact of intensive hydration on the incidence of post-ERCP pancreatitis: doubleblinded randomized controlled trial. Gastrointest Endosc 2016; 83: AB250

[49] Shaygan-Nejad A, Masjedizadeh AR, Ghavidel A et al. Aggressive hydration with Lactated Ringer's solution as the prophylactic intervention for postendoscopic retrograde cholangiopancreatography pancreatitis: A randomized controlled double-blind clinical trial. J Res Med Sci 2015; 20: 838-843

[50] NorthShore University Health System, Medical College of Wisconsin. et al. High volume lactated Ringer's solution and pancreatitis. Na- tional Library of Medicine (US); 2016: Available at: https://clinicaltrials.gov/show/NCT02050048 [Accessed December 21, 2016.]

[51] Mok SRS, Ho HC, Shah P et al. Lactated Ringer's solution in combination with rectal indomethacin for prevention of post-ERCP pancreatitis and readmission: a prospective randomized, double-blinded, placebo-controlled trial. Gastrointest Endosc 2017; 85: 1005-1013

[52] Harewood GC, Pochron NL, Gostout C]. Prospective, randomized, controlled trial of prophylactic pancreatic stent placement for endoscopic snare excision of the duodenal ampulla. Gastrointest Endosc 2005; 62: $367-370$

[53] Sofuni A, Maguchi H, Itoi T et al. Prophylaxis of post-endoscopic retrograde cholangiopancreatography pancreatitis by an endoscopic pancreatic spontaneous dislodgement stent. Clin Gastroenterol Hepatol 2007; 5: $1339-1346$

[54] Pan XP, Dang T, Meng XM et al. Clinical study on the prevention of post-ERCP pancreatitis by pancreatic duct stenting. Cell Biochem Biophys 2011; 61: 473-479

[55] Lee TH, Moon JH, Choi H] et al. Prophylactic temporary 3F pancreatic duct stent to prevent post-ERCP pancreatitis in patients with a difficult biliary cannulation: a multicenter, prospective, randomized study. Gastrointest Endosc 2012; 76: 578-585

[56] Kawaguchi Y, Ogawa M, Omata F et al. Randomized controlled trial of pancreatic stenting to prevent pancreatitis after endoscopic retrograde cholangiopancreatography. World J Gastroenterol 2012; 18: $1635-1641$

[57] Cha SW, Leung WD, Lehman GA et al. Does leaving a main pancreatic duct stent in place reduce the incidence of precut biliary sphincterotomy-associated pancreatitis? A randomized, prospective study Gastrointest Endosc 2013; 77: 209-216

[58] Olsson G, Lubbe J, Arnelo U et al. The impact of prophylactic pancreatic stenting on post-ERCP pancreatitis: A nationwide, register-based study. United European Gastroenterol J 2017; 5: 111 - 1118

[59] Dumonceau JM, Andriulli A, Elmunzer B] et al. Prophylaxis of postERCP pancreatitis: European Society of Gastrointestinal Endoscopy (ESGE) Guideline - updated June 2014. Endoscopy 2014; 46: 799-815

[60] Yokoe M, Takada T, Mayumi T et al. Japanese guidelines for the management of acute pancreatitis: Japanese Guidelines 2015. J Hepatobiliary Pancreat Sci 2015; 22: 405-432

[61] Akbar A, Abu Dayyeh BK et al. Rectal nonsteroidal anti-inflammatory drugs are superior to pancreatic duct stents in preventing pancreatitis after endoscopic retrograde cholangiopancreatography: a network meta-analysis. Clin Gastroenterol Hepatol 2013; 11: 778-783

[62] Medical University of South Carolina. Stent vs. indomethacin for preventing post-ERCP pancreatitis (SVI). National Library of Medicine (US); 2018: Available at: https://clinicaltrials.gov/ct2/show/ NCT02476279? cond=stent+vs.+indomethacin+for+preventing +post-ercp+pancreatitis\&rank=1 [Accessed March 21, 2018.]

[63] Cote GA. The end of prophylactic pancreatic duct stents? Proceed with caution and courage Clin Gastroenterol Hepatol 2014; 12: 528

[64] Cheng CL, Sherman S, Watkins JL et al. Risk factors for post-ERCP pancreatitis: a prospective multicenter study. Am J Gastroenterol 2006; 101: 139-147

[65] Cotton PB, Garrow DA, Gallagher J et al. Risk factors for complications after ERCP: a multivariate analysis of 11,497 procedures over 12 years. Gastrointest Endosc 2009; 70: $80-88$

[66] Freeman ML, DiSario JA, Nelson DB et al. Risk factors for post-ERCP pancreatitis: a prospective, multicenter study. Gastrointest Endosc 2001; 54: $425-434$ 